\title{
Taking sides with pain - lateralization aspects related to cerebral processing of dental pain
}

\author{
Mike Brügger ${ }^{*+}$, Dominik A. Ettlin ${ }^{2+}$, Michael Meier $^{1}$, Thierry Keller $^{3}$, Roger Luechinger $^{4}$, Ashley Barlow $^{5}$, \\ Sandro Palla', Lutz Jäncke' and Kai Lutz' \\ 1 Division of Neuropsychology, Department of Psychology, University of Zurich, Zurich, Switzerland \\ 2 Center for Dental Medicine, Clinic for Removable Prosthodontics, Masticatory Disorders and Special Care Dentistry, University of Zurich, Zurich, Switzerland \\ ${ }^{3}$ Automatic Control Laboratory, Swiss Federal Institute of Technology, Zurich, Switzerland \\ ${ }^{4}$ Institute of Biomedical Engineering, Swiss Federal Institute of Technology and the University of Zurich, Switzerland \\ ${ }^{5}$ GlaxoSmithKline, Consumer Healthcare, Weybridge, Surrey, UK
}

\section{Edited by:}

Alvaro Pascual-Leone, Beth Israel

Deaconess Medical Center/Harvard

Medical School, USA

Reviewed by:

Felipe Fregni, Spaulding Rehabilitation

Hospital, USA

Jean-Pascal Lefaucheur, Université

Paris XII, France

*Correspondence:

Mike Brügger, Research Group

"Cortical Pain," Neuropsychology,

Institute for Psychology, University of

Zurich, Binzmühlestrasse 14, CH-8050

Zurich, Switzerland.

e-mail:michael.bruegger@uzh.ch

${ }^{\dagger}$ Mike Brügger and Dominik A. Ettlin

have contributed equally to this study.
The current $\mathrm{fMRI}$ study investigated cortical processing of electrically induced painful tooth stimulation of both maxillary canines and central incisors in 21 healthy, right-handed volunteers. A constant current, 150\% above tooth specific pain perception thresholds was applied and corresponding online ratings of perceived pain intensity were recorded with a computerized visual analog scale during $\mathrm{fMRI}$ measurements. Lateralization of cortical activations was investigated by a region of interest analysis. A wide cortical network distributed over several areas, typically described as the pain or nociceptive matrix, was activated on a conservative significance level. Distinct lateralization patterns of analyzed structures allow functional classification of the dental pain processing system. Namely, certain parts are activated independent of the stimulation site, and hence are interpreted to reflect cognitive emotional aspects. Other parts represent somatotopic processing and therefore reflect discriminative perceptive analysis. Of particular interest is the observed amygdala activity depending on the stimulated tooth that might indicate a role in somatotopic encoding.

Keywords: toothache, fMRI, dominance, cerebral, amygdala, cerebral cortex, lateralization

\section{INTRODUCTION}

Brain structures consistently activated by noxious stimuli are: anterior cingulate cortex (ACC), insula, secondary somatosensory cortex (SII), lentiform nuclei, cerebellum, and thalamus. Less consistently, activation related to nociception has been reported for primary somatosensory cortex (SI), motor cortex (M1), premotor areas, and subcortical structures (Treede et al., 1999; Petrovic et al., 2000; Peyron et al., 2000; Bingel et al., 2002; Farrell et al., 2005). Functionally, these areas have been divided into a lateral and medial pain system and substantial evidence has emerged in support of this model (Albe-Fessard et al., 1985; Bushnell et al., 1999; Tracey and Mantyh, 2007), although alternative hypotheses have also been put forward (Apkarian et al., 2005; Craig, 2005; Mouraux and Iannetti, 2009).

Generally, the medial pain system composed of the insular cortex, anterior cingulate, and limbic structures is held responsible for processing emotional-affective and cognitive-behavioral pain aspects (Kulkarni et al., 2005; Wiech et al., 2006). The lateral pain system is attributed to sensory-discriminative components of pain and includes the lateral spinothalamic tract, the ventral posterolateral nucleus of the thalamus, and SI (Kenshalo Jr. et al., 1988; Bushnell and Duncan, 1989; Bushnell et al., 1999). In line with these functional attributes, one would expect to find evidence from experimental pain studies showing contralateral activation in this lateral system in response to unilateral noxious stimuli. Surprisingly, human imaging studies cannot consistently confirm activation in the lateral system in response to unilateral noxious stimuli. SI for example is only activated in approximately 50-75\% of all reports (Bushnell et al., 1999; Peyron et al., 2000; Apkarian et al., 2005; Farrell et al., 2005). Similarly, hard evidence is lacking for distinct contralateral hemispheric activation of other structures of the lateral pain system. One explanation may be that only few studies report on administering noxious stimuli to bilateral homologous body parts (Coghill et al., 1999, 2001; Bingel et al., 2002, 2003; Brooks et al., 2002; Youell et al., 2004; Symonds et al., 2006). The current study aimed at elucidating cortical spatial representation and hemispheric lateralization in response to dental nociception.

Ideally, lateralization aspects of pain were investigated by asynchronously applying bilateral noxious stimuli at graded distances to the body midline. This is readily realized by stimulation of multiple teeth as previously done (Ettlin et al., 2004, 2009). A possible interference by midline crossing of maxillary nerve endings is unlikely based on findings by Kemppainen et al. (2003). Jantsch et al. (2005) published the first brain fMRI investigation on tooth pain induced by electric stimulation. However, they stimulated one single tooth only as well as the ipsilateral dorsal hand. The results of their study suggest that brain processing of electrically evoked dental pain shows similarities as well dissimilarities compared to upper extremity mechanically induced pain. 
Based on the model of a lateral and medial pain system, we hypothesized that within the cortical pain circuitry, certain brain areas be activated dependent on the stimulation side and others showing lateralized or bilateral hemispheric activity independent of the side of stimulus application.

\section{MATERIALS AND METHODS PARTICIPANTS}

Twenty-one neurologically healthy subjects ( 8 female/13 male, age 20-44, all right-handed (Annett, 1970) with no dental pain experience during the preceding year participated in the experiment. Inclusion criteria required test teeth to be caries free, vital, and without attachment loss. Dental and periodontal pathologies were excluded by professional dental and radiographic examinations of maxillary teeth. Subjects received detailed information about the experimental procedure and provided written informed consent. The study was approved by the local ethics committee and was conducted according to the guidelines of the Declaration of Helsinki for treatment of experimental human subjects.

\section{EXPERIMENTAL MATERIAL}

Maxillary alginate impressions were taken from the subjects' dentitions for fabrication of soft dental acrylic splints. Four pairs of stainless steel electrodes were embedded in each individual dental splint opposite the labial and palatal surface center of the target teeth, namely maxillary canines and central incisors (Figure 1). They served as anode and cathode during electric stimulation. In order to minimize electric resistance during stimulation, a round piece of hydrogel (AG602-6, AMGEL Technologies, Lystrup, Denmark)

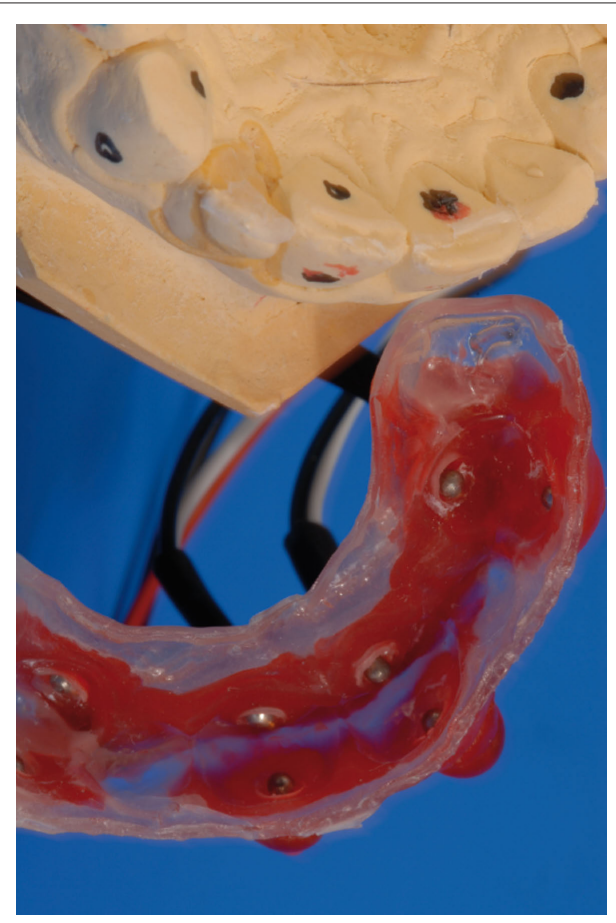

FIGURE 1 | Customized acrylic splint with carbon wires and stainless steel electrodes (fabricated for each subject). Electrodes were placed on the labial and oral face of the respective tooth. with $3 \mathrm{~mm}$ diameter was placed between the tooth and anode and cathode, respectively, and was covered with a thin layer of toothpaste (Signal Microgranuli, Unilever, Zug, Switzerland).

Electric stimulation was performed by means of the portable system Compex Motion System (Keller et al., 2002) and the experimental protocol was controlled by the Presentation software ${ }^{1}$ via parallel port using a self made interface. To avoid radiofrequency contamination of the stimulation current, specially shielded wires were used. For rating of the stimulus intensities within the MRI scanner, a computerized visual analog scale was used (COVAS; MEDOC, Haifa, Israel), with anchor points "no pain" on the left and "worst imaginable pain" on the right. This COVAS was projected onto a screen outside the scanner, and a mirror based deflection system enabled its visibility for the subjects.

\section{SENSORY TESTING PRIOR TO THE MR EXPERIMENT}

One to two weeks prior to the MR experiment, sensory testing with the tooth stimulation setup was performed in order to assess individual thresholds for sensory perception (SPT), pain perception (PPT), and pain tolerance (PTT) separately for each target tooth. The three thresholds were defined as the average ascending electric stimulus intensity out of three tests at which the subject reported sensation, pain, and PTT, respectively. We also questioned subjects whether single stimuli were felt distinctly in one test tooth only, which was acknowledged by all participants. Sequence of tooth stimulation was randomized between individuals.

For all tooth stimuli (threshold determination and fMRI stimulation protocol) biphasic pulse forms of $1 \mathrm{~ms}$ duration were applied on both maxillary canine and medial incisors with interstimulus intervals randomized between 7.5 and $10 \mathrm{~s}$.

\section{fMRI DATA ACQUISITION AND STIMULATION PROTOCOL}

Within 1-2 weeks after sensory testing, subjects underwent the fMRI protocol in a Philips 3-T Achieva system (Philips Medical Systems, Best, The Netherlands) at the same time of day as threshold determination was performed, since evidence indicates a diurnal association of somesthetic perception (Fillingim and Ness, 2000; Sessle, 2000; Wiesenfeld-Hallin, 2005). Subjects were placed in the scanner in a supine position and their individual SPT and PPT were re-tested inside the scanner to exclude changes related to the experimental setting. No significant differences were observed [analysis of variance (ANOVA), Greenhouse-Geisser corrected, $F=1.653$, $\left.p=0.187, \eta^{2}=0.076\right]$. The fMRI stimulation protocol consisted of 40 constant stimuli per tooth applied in randomized order to the four teeth with an intensity $150 \%$ of the tooth specific PPT. Pain intensity ratings were used to control for differences in perceived pain intensity among tested teeth. For each tooth subjects were requested to rate the pain intensity of 10 randomly selected stimuli ( $25 \%$ of all stimuli applied). For those stimuli to be rated, the VAS appeared directly after stimulus delivery, and subjects were offered $5 \mathrm{~s}$ for pain intensity rating. For the remaining $75 \%$ of trials, the stimulus was followed by a fixation cross on the screen. We decided not to have every stimulus rated in order to minimize motion artifacts and other rating influences (Schoedel et al., 2008). All scans followed by a rating were therefore excluded from further fMRI analysis. The experimental run lasted approximately $23 \mathrm{~min}$.

${ }^{1}$ www.neurobs.com/presentation 
For the functional scans, a blood oxygen level dependent (BOLD) sensitive single-shot gradient echo planar imaging sequence was used with 33 axial slices, covering the entire cerebrum and cerebellum, using an eight-channel receive-only head coil. Parameters: echo time $=30 \mathrm{~ms}$, flip angle $=75^{\circ}$, repetition time $=2500 \mathrm{~ms}$, slice thickness $=4 \mathrm{~mm}$, inter-slice gap $=0 \mathrm{~mm}$, field of view $=230 \mathrm{~mm}$, and matrix size in plane $=128 \times 128$, resulting in a voxel size of $1.72 \mathrm{~mm} \times 1.72 \mathrm{~mm} \times 4 \mathrm{~mm}$. Three "dummy" scans were first acquired to reach steady state magnetization and discarded. A total of 180 high-resolution T1 weighted axial slices (spoiled gradient echo) were acquired with $\mathrm{TR}=20 \mathrm{~ms}$, flip angle $=20^{\circ}$, voxel size $=$ $0.98 \mathrm{~mm} \times 0.98 \mathrm{~mm} \times 1.02 \mathrm{~mm}$, FOV $=24 \mathrm{~cm}$, matrix $=256 \times 192$, which were used as an underlay for individual functional maps.

\section{DATA ANALYSIS}

Individual PPT thresholds were analyzed with respect to differences between the laboratory and fMRI condition in a repeated measures ANOVA (RM-ANOVA), with the factors "location" and "tooth." A separate ANOVA with mean COVAS ratings per tooth as dependent variable, "tooth" as within-subject factor and "gender" as between-subject factor was calculated to check whether within each subject pain intensity and PPT varies between the stimulated teeth.

Functional image analysis was done using the SPM5 software package $^{2}$ running on MatLab R2007a (MathWorks, Natick, USA). In a first step, spatial realignment and reslicing to the first image in the series as reference was performed [detected movement did not exceed $1.5 \mathrm{~mm}$ (translational) or $1^{\circ}$ (rotational) compared with the reference image]. For studying group effects, data were normalized to the MNI template brain (Evans et al., 1993) followed by smoothing with a Gaussian kernel of $6 \mathrm{~mm}$ (FWHM) and scaled to the global mean intensity. A general linear model (GLM) was setup and estimated. Differences between stimulation and baseline were transformed into color-coded T-maps for each voxel and superimposed onto the MNI single-subject-T1 brain. Corrected data [family wise error (FWE); Worsley et al., 1996] with $p<0.01$ are reported in the general cortical activation section. Regions of interest (ROI) were defined, based on images provided by the "WFU-Pickatlas" (Lancaster et al., 1997, 2000; Maldjian et al.,

${ }^{2} \mathrm{http}: / /$ www.fil.ion.ucl.ac.uk/spm
2003) for areas selected from pain literature reviews (Peyron et al., 2000; Farrell et al., 2005): postcentral gyrus, thalamus, amygdala, supramarginal (BA 40), preparietal (BA 5), and superior parietal (BA 7) areas, subcentral area (BA 43), cerebellum (anterior and posterior lobe), the supplemental motor area (BA 6), frontomedial area (BA 46), and frontopolar (BA 10) areas, hippocampus, parahippocampus, caudate, putamen, pallidum, and the brainstem. Two exceptions were applied: the "insula-ROI" provided by the WFUPickatlas was divided into three parts (anterior, medial, and posterior) according to Brooks et al. (2002), since several reports suggest a complex anatomical (Varnavas and Grand, 1999) and functional (Coghill et al., 1999; Brooks et al., 2002, 2005; Symonds et al., 2006) fragmentation within the insula. To take into account the functional complexity of the cingulate cortex, we subdivided this structures based on Vogt (2005). The numbers of activated voxels, mean- and maximum activation were calculated within each ROI.

Data were then analyzed using SPSS for Windows (Release 14.0.0, SPSS Inc., Chicago, IL, USA). A RM-ANOVA with "hemisphere" and "side of stimulation" as within-subjects-factors was performed for the ROIs. Main effects for factor "hemisphere" as well as interaction between factors "hemisphere" and "side of stimulation" were analyzed. For RM-ANOVAs, results were Greenhouse-Geisser corrected for non-sphericity if applicable.

\section{RESULTS}

\section{PSYCHOPHYSICS}

Mean stimulus intensities of the general study population during the scanning procedure demonstrated a significant within-subjects effect $(F=3.45, p=0.02)$ ranged from 20.76 to $25.24 \mathrm{~mA}$ across the four teeth, whereas respective ratings ranged from 46.9 to 49.1 but showed no significant differences $(F=0.48, p=0.70)$. According to gender related differences, we found a trend in the interaction gender $\times$ stimulus intensities $\left(F=2.74, \eta^{2}=0.13, p=0.051\right)$ but no interaction according to the gender $\times$ rating interaction with $F=0.87, \eta^{2}=0.04$, and $p=0.46$ (for detailed information please see Table 1 ).

In the overall study population, post hoc t-test on stimulus intensity revealed a significant difference between right central incisor and right canine $(t=3.82, p=0.001)$ as well as between right central incisor and left canine $(t=2.83, p=0.01)$. An additional one-way ANOVA exploring possible gender differences showed a significant difference between the stimulus intensities of the left central

Table 1 | Mean stimulus intensities and related mean ratings during fMRI in the overall study population and differentiated by gender.

\begin{tabular}{|c|c|c|c|c|}
\hline & \multicolumn{2}{|c|}{ Right } & \multicolumn{2}{|c|}{ Left } \\
\hline & Canine & Central incisor & Canine & Central incisor \\
\hline \multicolumn{5}{|l|}{ OVERALL $(N=21)$} \\
\hline Stimulus intensities (mA) & $25.2 \pm 10.3$ & $20.8 \pm 11.3$ & $24.8 \pm 11.5$ & $23.9 \pm 13.1$ \\
\hline COVAS ratings (0-100) & $46.7 \pm 18.5$ & $48.0 \pm 19.7$ & $45.5 \pm 19.0$ & $46.9 \pm 18.3$ \\
\hline \multicolumn{5}{|l|}{ FEMALE $(N=8)$} \\
\hline Stimulus intensities (mA) & $21.9 \pm 9.6$ & $17.0 \pm 7.5$ & $18.8 \pm 7.5$ & $15.8 \pm 5.8$ \\
\hline COVAS ratings (0-100) & $38.0 \pm 13.3$ & $43.5 \pm 20.2$ & $39.5 \pm 18.8$ & $41.5 \pm 17.6$ \\
\hline \multicolumn{5}{|l|}{ MALE $(N=13)$} \\
\hline Stimulus intensities (mA) & $27.3 \pm 10.6$ & $23.1 \pm 12.8$ & $28.5 \pm 12.1$ & $28.9 \pm 13.9$ \\
\hline COVAS ratings (0-100) & $52.1 \pm 19.6$ & $50.8 \pm 19.6$ & $49.1 \pm 18.8$ & $50.2 \pm 18.6$ \\
\hline
\end{tabular}


incisor $(F=6.30, p=0.2)$ and a trend with respect to the left canine $(F=4.17, p=0.55)$. All other comparisons reached no significant level. All values are listed with respective standard deviations.

\section{HEMODYNAMIC RESPONSES ACROSS THE ENTIRE BRAIN AND WITHIN REGIONS OF INTEREST}

Group activation brain maps (stimulation versus baseline) are displayed in Figure $\mathbf{2}$ and specified in Table A1 in Appendix (as we focus on the lateralization analyses, we disclaim from describing this patterns here more extensively). All ROIs investigated showed significant activation compared to baseline, namely postcentral gyrus, thalamus, preparietal (BA 5), and superior parietal (BA 7) areas, supramarginal (BA 40) and subcentral areas (BA 43), anterior, medial, and posterior insula, amygdala, hippocampus, parahippocampus, both cerebellae (anterior and posterior lobe), caudate, putamen, pallidum, supplementary motor (BA 6), frontomedial (BA 46), and frontopolar areas (BA 10) the subdivisions of the cingulate gyrus [PCC, pMCC, aMCC, pregenual ACC (pACC), sACC, and the brainstem; Table 2].

\section{LATERALIZATION EFFECTS BASED ON REGIONS OF INTEREST ANALYSIS}

(1) There are no ROIs demonstrating a significant effect for "side of stimulation.”

A

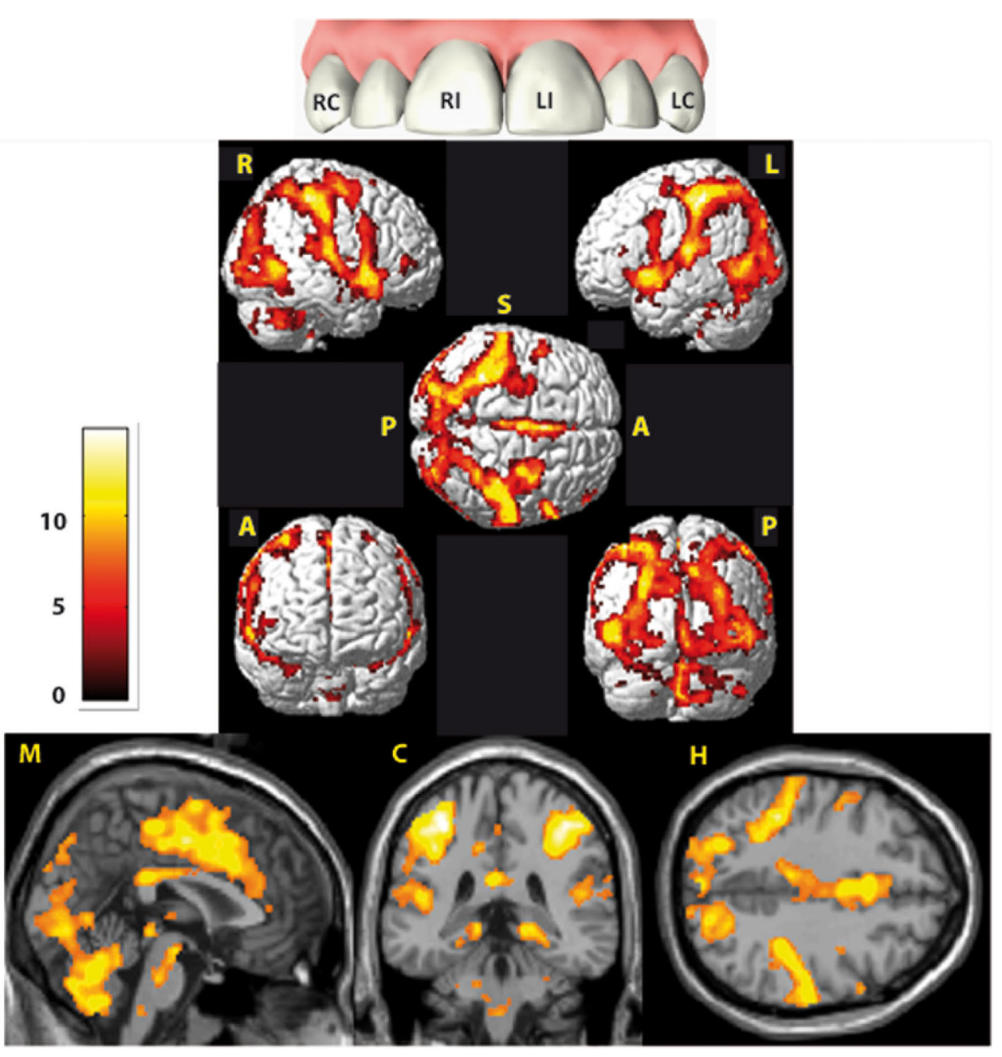

B
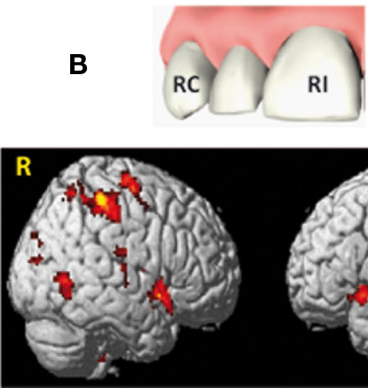

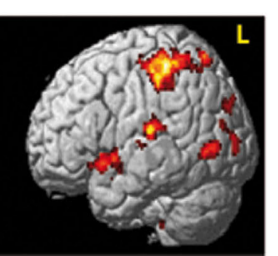

FIGURE 2 | Cortical areas activated by electric tooth stimulation across all four teeth (A) and with respect to both right teeth (RI and $\mathrm{RC})$ and both left teeth (LI and $L C$ ) respectively $(\mathbf{B}, \mathbf{C})$. Activity is projected onto the single-subjectMNI-template. Indicators at the rendered brains stand for the views: $\mathrm{R}$, from

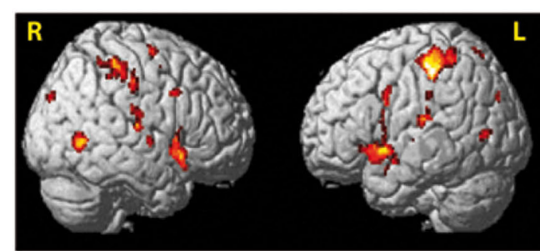

right; L, from left; $S$, from superior; $A$, from anterior; $P$, from posterior, all brain figures are in neurological orientation. Slices from left to right: midsagittal (M), coronal (C) at $Y=-36$ and horizontal $(\mathrm{H})$ at $Z=54$. Data are corrected for multiple comparison (FWE) $p=0.01$ with an extended threshold of 10 voxels. 
Table 2 | Activation statistics in the selected regions of interest (see Materials and Methods) based on group analysis pooled across all four teeth.

\begin{tabular}{|c|c|c|c|c|c|}
\hline Anatomical description & Hemisphere & Cluster size & $\begin{array}{l}\text { MNI coordinates } \\
\text { (max } T \text { voxel) }\end{array}$ & $\begin{array}{l}\text { Voxel } p \\
\text { (FWE-corrected) }\end{array}$ & $\begin{array}{l}\text { Voxel } T \\
(\max T)\end{array}$ \\
\hline Postcentral gyrus (SI) & $L$ & 970 & $-38-3654$ & 0.000 & 14.74 \\
\hline \multirow[t]{2}{*}{ Thalamus } & $L$ & 281 & $-10-208$ & 0.000 & 11.04 \\
\hline & $\mathrm{R}$ & 275 & $14-1610$ & 0.000 & 9.94 \\
\hline Preparietal area (BA 5) & $\mathrm{L}$ & 46 & $-34-4462$ & 0.000 & 11.13 \\
\hline \multirow[t]{2}{*}{ Superior parietal area (BA 7) } & $\mathrm{L}$ & 502 & $-22-6662$ & 0.000 & 10.78 \\
\hline & $\mathrm{R}$ & 387 & $16-7834$ & 0.000 & 9.60 \\
\hline \multirow[t]{2}{*}{ Supramarginal area (BA 40) } & $\mathrm{L}$ & 586 & $-40-3658$ & 0.000 & 13.66 \\
\hline & $\mathrm{R}$ & 374 & $50-3050$ & 0.000 & 12.16 \\
\hline \multirow[t]{2}{*}{ Subcentral area//SII (BA 43) } & $\mathrm{L}$ & 9 & $-52-1816$ & 0.000 & 6.69 \\
\hline & $\mathrm{R}$ & 36 & $66-1620$ & 0.000 & 10.07 \\
\hline \multirow[t]{2}{*}{ Posterior insula } & $L$ & 52 & $-44-142$ & 0.000 & 7.93 \\
\hline & $\mathrm{R}$ & 41 & $42-12-8$ & 0.000 & 7.92 \\
\hline \multirow[t]{2}{*}{ Amygdala } & $L$ & 90 & $-200-12$ & 0.000 & 7.54 \\
\hline & $\mathrm{R}$ & 72 & $262-20$ & 0.000 & 8.37 \\
\hline \multirow[t]{2}{*}{ Hippocampus } & $L$ & 79 & $-20-24-10$ & 0.000 & 10.57 \\
\hline & $\mathrm{R}$ & 48 & $18-360$ & 0.000 & 8.62 \\
\hline \multirow[t]{2}{*}{ Parahippocampus } & $\mathrm{L}$ & 47 & $-24-26-16$ & 0.000 & 7.80 \\
\hline & $\mathrm{R}$ & 144 & $16-38-6$ & 0.000 & 8.88 \\
\hline \multirow[t]{2}{*}{ Cerebellum anterior lobe } & $L$ & 617 & $-34-58-34$ & 0.000 & 9.21 \\
\hline & $\mathrm{R}$ & 1017 & $2-62-26$ & 0.000 & 9.96 \\
\hline Cerebellum posterior lobe & $\mathrm{L}$ & 617 & $-2-72-38$ & 0.000 & 9.54 \\
\hline \multirow[t]{2}{*}{ Supplementary motor area (BA 6) } & $\mathrm{L}$ & 631 & -2648 & 0.000 & 11.68 \\
\hline & $\mathrm{R}$ & 421 & 2846 & 0.000 & 10.71 \\
\hline \multirow[t]{2}{*}{ Frontomedial area (BA 46) } & $L$ & \multicolumn{4}{|c|}{ No suprathreshold cluster with this conservative statistic level } \\
\hline & $\mathrm{R}$ & 7 & 52426 & 0.000 & 7.05 \\
\hline Frontopolar area (BA 10) & $L$ & No suprathres & er with this conservat & ic level & \\
\hline & $\mathrm{R}$ & 1 & 52420 & 0.000 & 6.41 \\
\hline PCC & $L$ & 280 & $-8-2844$ & 0.000 & 10.70 \\
\hline & $\mathrm{R}$ & 108 & $2-2852$ & 0.000 & 9.02 \\
\hline $\mathrm{pMCC}$ & $L$ & 249 & $-2-648$ & 0.000 & 11.33 \\
\hline & $\mathrm{R}$ & 259 & $8-846$ & 0.000 & 11.93 \\
\hline aMCC & $L$ & 521 & -2640 & 0.000 & 10.37 \\
\hline & $\mathrm{R}$ & 480 & 21638 & 0.000 & 9.83 \\
\hline pACC & $\mathrm{L}$ & 103 & -23218 & 0.000 & 6.90 \\
\hline & $\mathrm{R}$ & 20 & 23420 & 0.000 & 6.75 \\
\hline sACC & $L$ & No suprathres & er with this conservat & ic level & \\
\hline & $\mathrm{R}$ & No suprathres & er with this conservat & ic level & \\
\hline Brainstem & $\mathrm{L}$ & 47 & $-2-34-50$ & 0.000 & 6.83 \\
\hline & $\mathrm{R}$ & 69 & $2-26-30$ & 0.000 & 7.82 \\
\hline
\end{tabular}

A small volume correction was performed with the regions of interests as search volume. Described are cluster size, MNI coordinates of the maximally activated voxel with the respective $p$ - and $t$-values. Data are family wise error (FWE) corrected $(p<0.01$, extent voxel threshold $k=10$ ). 
(2) There were several ROIs that were strongly activated on one hemisphere irrespective of the side of stimulation. Both, anterior and posterior cerebellar lobes demonstrated a stronger right hemispheric effect. A stronger left hemispheric effect was found in putamen, pACC, supramarginal area (BA 40), and parahippocampus (Table 3; Figure 3).

(3) There was one region, namely the subcentral area (BA 43), in which "hemisphere" showed a stronger right sided effect as well as an interaction with the factor "side of stimulation" (Table 3; Figure 3). This laterality effect was observed especially after left sided stimulation.

(4) There were several regions in which no main effect but an interaction between "hemisphere" and "side of stimulation" was observed (Table 3; Figure 3). Postcentral gyrus (SI), posterior insula, thalamus, and amygdala all showed a hemispheric dominance contralateral to the stimulation side.

\section{DISCUSSION}

The aim of this study was to elucidate cortical spatial representation and hemispheric lateralization in response to noxious electric dental stimulation. Findings reveal robust brain activation in areas previously shown to be involved in pain processing.

Focusing on lateralization aspects, we categorize the findings into three groups: (1) structures exhibiting hemispheric lateralization irrespective of side of stimulation, (2) structures showing activation dominance contralateral to the side of stimulation without hemispheric lateralization, and (3) structures demonstrating not only hemispheric lateralization, but also dependency on side of stimulation. In the following, we discuss these findings in detail.

\section{HEMISPHERIC LATERALIZATION IRRESPECTIVE OF SIDE OF STIMULATION}

We found evidence for hemispheric lateralization in six brain areas irrespective of side of stimulation. The anterior and posterior cerebellar lobes as well as the parahippocampus demonstrate a stronger right hemispheric effect, whereas a stronger left hemispheric effect was observed in putamen, pregenual cingulate cortex, and supramarginal area (BA 40).

Pain related cerebellar activity has been consistently demonstrated (reviewed in Peyron et al., 2000; Apkarian et al., 2005; Farrell et al.,2005) and several suggestions are published in order to explain this often robust activity (see e.g., Saab and Willis, 2003). Evidence for direct and/or collateral trigeminal input to cerebellar structures is provided by animal studies (Snyder et al., 1978; Dietrichs and Walberg, 1987; Patrick and Robinson, 1987; Saab et al., 2001; Bukowska et al., 2006; Holtzman et al., 2006). Findings revealed that trigeminal brainstem nuclei interpolaris, oralis, and principalis project predominantly ipsilateral to cerebellar regions. Taken together, cerebellar cortices receive mostly ipsilateral and to a lower extend, bilateral fibers from several trigeminal brainstem nuclei (detailed summarized by Dietrichs and Walberg, 1987). Recent work by Borsook et al. (2008) provides an overview of 28 studies with cerebellar activation in acute experimental pain using fMRI and PET. Bilateral activity is described in 15 , ipsilateral activity in 10 , and contralateral activity in 3 of them. This is an astonishing observation as most of the reviewed investigations stimulated the upper extremities unilaterally. Considering the anatomical perspective provided by animal research, one would expect a predominantly ipsilateral and to a smaller extent, bilateral activation. They also summarize own research on investigating specifically noxious and non-noxious thermal heat and brush stimuli applied to the maxillary division of the face in healthy and neuropathic pain patients. Summarized, noxious heat evoked predominantly contralateral activation in both groups, while brush evoked more ipsilateral cerebellar activity. Based on their observations a "dichotomy of innocuous stimuli/sensorimotor cerebellum activation versus noxious experience/cognitive/ limbic cerebellum activation" was suggested.

Our data show a right-lateralized effect in both, anterior and posterior cerebellum as well as in the parahippocampus. Schmahmann and Pandya (1997) as well as Manto (2006) describe outputs to

Table 3 | Repeated measures ANOVA results of the region of interest analysis.

\begin{tabular}{lll}
\hline Anatomical description & Main effect “hemisphere" $\left[\boldsymbol{F ( \eta ^ { 2 } ) \boldsymbol { p } ]}\right.$ & $\begin{array}{l}\text { Interaction effect "to } \\
{\left[\boldsymbol{F}\left(\boldsymbol{\eta}^{2}\right) \boldsymbol{p}\right]}\end{array}$ \\
\hline Thalamus & & $11.038(0.356) \mathbf{0 . 0 0 3}$ \\
Postcentral gyrus (SI) & $0.028(0.001) 0.870$ & $12.928(0.393) \mathbf{0 . 0 0 2}$ \\
Posterior insula & $0.876(0.042) 0.360$ & $4.564(0.186) \mathbf{0 . 0 4 5}$ \\
Amygdala & $0.003(0.000) 0.959$ & $23.163(0.537) \mathbf{0 . 0 0 0}$ \\
Subcentral area (BA 43) & $3.615(0.153) 0.072$ & $12.899(0.392) \mathbf{0 . 0 0 2}$ \\
Preparietal area (BA 5) & $17.723(0.470) \mathbf{0 . 0 0 0}$ & $3.008(0.131) 0.098$ \\
Cerebellum (posterior lobe) & $1.219(0.057) 0.283$ & $1.349(0.063) 0.259$ \\
Cerebellum (anterior lobe) & $18.814(0.485) \mathbf{0 . 0 0 0}$ & $1.942(0.089) 0.179$ \\
Parahippocampus & $4.546(0.185) \mathbf{0 . 0 4 6}$ & $1.417(0.066) 0.248$ \\
Supramarginal area (BA 40) & $6.628(0.249) \mathbf{0 . 0 1 8}$ & $1.654(0.076) 0.213$ \\
Pregenual anterior cingulate (pACC) & $7.191(0.264) \mathbf{0 . 0 1 4}$ & $0.771(0.037) 0.515$ \\
Anterior medial cingulate (aMCC) & $13.934(0.411) \mathbf{0 . 0 0 0}$ & $0.507(0.025) 0.679$ \\
Putamen & $4.271(0.176) 0.052$ & $0.718(0.035) 0.407$ \\
Supplementary motor area (BA 6) & $7.213(0.265) \mathbf{0 . 0 1 4}$ & $0.357(0.018) 0.557$
\end{tabular}

Only the significant and ( $p<0.05)$, trend-like interactions $(p<0.10)$ are shown (see Figure 3 for illustration). Main effect "tooth" is not shown, as there is neither a significant nor a trend within that factor. F, F-value, $p, p$-value, $\eta^{2}$, proportion of the variability in the dependent measure that is attributable to a factor. Bold indicates $p<0.5$, italicized indicates a trend $(p<0.1)$. 


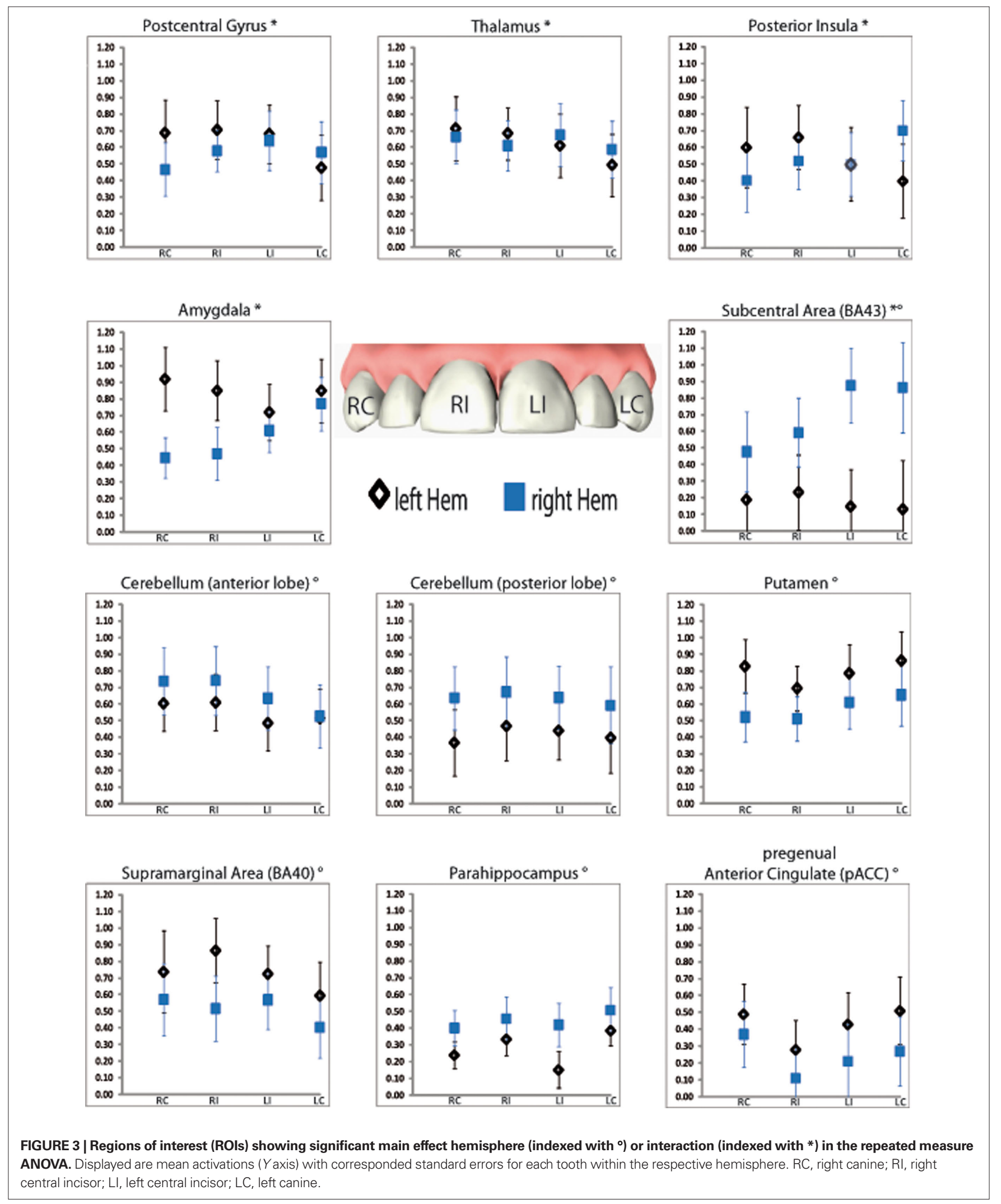

numerous (limbic) structures, among them; hippocampal complex, amygdala, thalamic nuclei, hypothalamus, and the periaqueductal gray. Based on these connections, the cerebellum has also been called "modulator of different neurologic functions," thus directly influencing sensory, but also emotional and cognitive processing (Allen et al., 2005; Ito, 2008). 
The role of the basal ganglia in processing nociceptive information is still debated despite their robustly observed involvement shown in human studies (Coghill et al., 1999, 2001; Apkarian et al., 2005) as well as in animal research (Chudler, 1998). Neuroanatomical evidence reveals afferents from several subdivisions of the cerebral cortex (including neocortical and cingulate cortex), thalamic nuclei, cerebellum, the amygdala, parabrachial area, and dorsal raphe nucleus (Chudler and Dong, 1995; Downar et al., 2003). Although the main role of the basal ganglia is often related to sensorimotor integration and thus adaptation of motor responses to noxious stimuli, their involvement in other dimensions of pain processing cannot be excluded. The review of Chudler and Dong (1995) provides strong evidence for a functional involvement of the basal ganglia in both, direct innocuous and noxious somatosensory processing. Supporting this finding, Coghill et al. (1999) pointed out the role of the putamen and globus pallidus (bilateral) in processing of human pain intensity and Scott et al. (2006) linked the role of the putamen to anticipatory mechanisms. Publications of several other investigations suggest cerebellar and basal ganglia processing to depend on cognitive functions (Akshoomoff and Courchesne, 1992; Schmahmann and Pandya, 1997; Schmahmann and Caplan, 2006). However, based on present literature no evidence emerges regarding lateralization of cognitive functions in these areas. Therefore, we do not assume that leftlateralization found in our data indicates cognitive involvement, but rather reveals motor functions, many of which are known to be lateralized to the motor dominant hemisphere. This interpretation is up for debate as two previous studies revealed certain aspects of hemispheric dominance to be independent of handedness for noxious and non-noxious somatosensory stimulation (Jung et al., 2003; Schlereth et al., 2003).

Focusing on significantly activated cingulate cortex subdivisions (PCC, pMCC, aMCC, pACC, and sACC) we found a left hemispheric lateralization in the pACC and a trend toward left-lateralization in the aMCC, but no lateralization in the more posterior divisions. Current literature indicates that pACC is associated with engaging in positively valenced events and is linked with the amygdala's lateral basal and accessory basal nuclei, whereas the aMCC contains the rostral cingulate motor area (Vogt, 2005). Based on their findings, Büchel et al. (2002) concluded that a main function of the ACC's subdivisions is to integrate a wide range of pain relevant information and to generate adequate responses. However, considering pain related investigations, distinct lateralization aspects of ACC subdivisions have to date not been in the focus of interest. In line with its functional attributes (selection of adequate reactions), the aMCC activation pattern found in our study points toward involvement in motor components of nociception, as seen for cerebellum and putamen (Vogt, 2005).

The left-lateralization effect noticed in the supramarginal area (BA 40) may also relate to a functional role of this structure in sensorimotor integration (Serrien et al., 2006), or a specialization for the detection of behaviorally relevant stimuli (Corbetta and Shulman, 2002).

Even if the stimuli may not be interpreted by subjects as potentially dangerous, pain is inherently salient (Legrain et al., 2009). Conform to Farrer et al. (2008) we favor an interpretation that the left lateralized activation within the supramarginal area is related to the analysis and integration of body-related nociceptive sensa- tions in contrast to right-lateralized parietal cortex activity which is thought to mediate the analysis and integration of body-related visual and painless somatosensory information.

With respect to the finding that parahippocampus shows predominantly right sided BOLD responses to dental nociceptive stimuli, the function of this structure may also be described in the context of novelty detection theories, as suggested before by Bingel et al. (2002) and Ploghaus et al. (2000) and corroborated by Strange and Dolan (2006) with fear related stimuli.

\section{STRUCTURES WITH PREDOMINANT CONTRALATERAL ACTIVATION}

We found evidence in five brain areas that reveal activation dominance contralateral to the side of stimulation: SI, thalamus, posterior insula, amygdala, and subcentral area (BA 43). Subcentral area additionally demonstrates hemispheric lateralization and will be discussed later.

Contralateral activation is closely linked to somatotopic encoding. Yet, unresolved questions exist as to lateralization aspects in cortical structures like SI, SII, thalamus, and posterior insula. To address this topic was one of the aims of the present study. Previously, Bingel et al. (2003) have investigated lateralized brain activity in response to noxious stimuli in SI, SII, insula, and thalamus and found contralateral bias in all these four areas. Although stimulation of either hand evoked bilateral activation of anterior and posterior insular regions, a contralaterally biased response was found for the posterior parts of the insula bordering SII. Similar findings were reported by Brooks et al. (2002) who applied noxious thermal stimuli to both hands. Again, activation in insular posterior parts was dependent on the site of stimulation, whereas this dependency was absent in more anterior insular areas and SII. Interestingly, activation was absent in thalamus and SI. If activation in the thalamus is reported, then mostly contralateral but also often bilateral (Peyron et al., 2000) although, more recently, Kulkarni et al. (2005) reported ipsilateral, but no contralateral thalamus activity.

Our electric dental stimulation data show robustly that SI is activated bilaterally with a significant predominance contralateral to the stimulus application side. The same findings hold true for thalamus, and posterior insular cortex (Figure 3). We thus confirm the functional role of these cortical areas in topographic stimulus encoding.

Possibly, lateralized activation of areas could be caused by evasive or protective motor action dependent on the site of stimulation. However, this unlikely explains the present data, since withdrawal and orientation responses have been shown to predominantly activate cingulate cortex subdivisions (Vogt, 2005; Peyron et al., 2007) and cerebellum (Dimitrova et al., 2003) but not SI, thalamus, posterior insula, amygdala, or subcentral area (BA 43).

The amygdala's involvement in various forms of conditioned hypoalgesia and analgesia has been well established in several animal studies (e.g., Crown et al., 2000; Neugebauer and Li, 2002, 2003; Neugebauer et al., 2004). Lesion studies, specifically of the latero-capsular amygdaloid nucleus (also termed "nociceptive amygdala") demonstrated reduced or completely abolished conditioned behavior (Watkins et al., 1998). Inconsistent amygdala activation in response to nociceptive and other aversive stimuli in humans is frequently reported (Baas et al., 2004; Phan et al., 2004; RempelClower, 2007; Tracey and Mantyh, 2007). Why amygdala activation appears robustly in response to noxious dental stimulation in 
comparison to stimulation of other body parts (Peyron et al., 2000; Apkarian et al., 2005; Farrell et al., 2005) can only be speculated. One possible explanation is that the amygdala has proven relevant for emotional conditioning (Büchel et al., 1999; Büchel and Dolan, 2000; Cardinal et al., 2002) and thus, a unique emotional salience of dental pain could explain our findings. However, it must be noted that the emotional value of the applied stimuli has not been directly controlled for. Stimulus conditioning and (missing) previous dental pain experiences could both contribute to an assumed peculiarity of dental pain. Alternatively dental pain may involve different processing pathways (trigeminal versus spinal). Future investigations need to further elucidate this topic.

Lateralization of amygdala activation shows an inconsistent picture. Among human neuroimaging studies, none described a clearly lateralized activation dependent on the stimulation side (e.g., Bingel et al., 2002; Bornhovd et al., 2002). The present data show that BOLD signal in the amygdala is stronger contralateral than ipsilateral to the side of stimulation. To the best of our knowledge, this has previously not been shown in pain studies nor in investigations on emotion. Regarding the latter, Baas et al. (2004) pointed out that there is no stimulation side dependent amygdala lateralization effect across 54 studies analyzed by them. One has of course to consider different paradigms and also different statistical approaches which hamper an adequate conclusion so far. Our approach of analyzing mean activations by a RM-ANOVA provides some evidence toward possible somatotopic related encoding properties. Previous studies may have missed a lateralization effect in the amygdala due to less salient stimuli and/or bigger voxel sizes (introducing greater partial volume effects and hence reduced statistical power).

Interestingly, contrary to previous reports our data do not indicate lateralization of brainstem activity. We propose that this is due to methodological reasons. Without applying special imaging techniques, brainstem activity is often severely masked by movement artifacts stemming from pulsation movements of the A. carotis. Correction of these artifacts involves, e.g., cardiac triggering, which we did not apply for sake of greater power in the remaining regions. Methods to deal with physiological artifacts post hoc (see e.g., Harvey et al., 2008) were also not applicable due to missing cardiac and respiratory information. Thus we argue that brainstem effects are likely to be missed in our study which should not give rise to suspicion regarding the effects found.

\section{STRUCTURES SHOWING HEMISPHERIC DOMINANCE AND PREDOMINANT CONTRALATERAL ACTIVATION}

The subcentral area (BA 43) shows significant lateralization to one hemisphere (main effect "hemisphere") and also significant enhanced activation contralateral to the stimulus. Interestingly, this area is not frequently reported in pain studies. Subcentral area (BA 43) is located at the ventral end of the pre/postcentral gyri and the bank of the lateral sulcus and also delineated as SII. Its rostral and caudal borders are neighbored by both, the anterior and posterior subcentral sulci. Its distinction from surrounding areas is based on its specific cytoarchitectonic features already observed by Brodmann (Eickhoff et al., 2006, 2007).

Only few human studies explicitly reported lateralized activation within BA 43 in response to noxious stimulation. Becerra et al. (2001) noted right-lateralized activation in BA 43 in response to noxious thermal hand stimulation, but this result was not addressed in the discussion. Focusing on idiopathic chronic low back pain, Giesecke et al. (2004) found bilateral activation in BA 43 and discussed it as being part of the SII. In a simultaneous EEG-fMRI investigation, Christmann et al. (2007) reported bilateral activation within BA 43 and also delineated it as being part of SII. However, in none of these studies, activity within BA 43 was further interpreted by the authors.

The present data showed a strong hemodynamic response within BA 43, with a significant interaction effect between stimulated tooth and hemisphere (activity is predominantly contralateral to the stimulus) as well as a main effect toward the right hemisphere (Table 3; Figure 3). This distinct right-lateralized activation is eyecatching and the present data may shed new light on the role of this structure, since the activation pattern is quite different from other parts of SII. Strong anatomical connections between the subcentral area and premotor cortices, as well as posterior parietal area (Cipolloni and Pandya, 1999) place the subcentral area (BA 43) in an ideal position for multimodal sensorimotor integration. Such a role has long been suggested for mammals (Krubitzer, 1996) and more recently for humans (Disbrow et al., 2000).

Although our results point toward a specialized somatosensory encoding function with a possible role in sensorimotor integration, it may be premature to speculate on the specific role of BA 43 within the pain circuitry.

\section{STUDY LIMITATIONS}

A full understanding of brain activations in response to painful stimuli is inherently limited by the complexity of the multidimensional pain experience. Some brain activity patterns may not necessarily be directly involved in pain processing, but rather relate to aspects of alertness and/or orientation responses. Namely parieto-occipital activation clusters may be interpreted this way. The human pain experience implies orientation toward pain and toward options to relieve it. Some brain activity may thus not be directly linked to the pain experience itself. Furthermore, as the intensities of all stimuli were above the pain threshold, purely somatosensory processes cannot be controlled for and thus it cannot be excluded that some brain activities may reflect somatosensory aspects of the stimulation. Finally, although all subjects located their pain to the stimulation tooth, we are unable to report on the fiber subpopulations involved in pain transmission.

\section{CONCLUSION}

Electrically evoked dental pain activates cortical areas typically described in spinal pain studies. Yet, robust activation can be observed in additional areas, namely the amygdala. Besides previously known lateralization effects, hemispheric lateralization irrespective of side of stimulation were observed in subdivisions of the ACC (aMCC and pACC). Predominant contralateral activation in the posterior insular cortex and the amygdala points toward their possible involvement in somatotopic encoding of noxious stimuli, in addition to other, previously described functions.

\section{ACKNOWLEDGMENTS}

This work was supported by GlaxoSmithKline, Division of Consumer Healthcare, the Swiss Dental Association, and the Swiss Society for Endodontology. 


\section{REFERENCES}

Akshoomoff, N. A., and Courchesne, E. (1992). A new role for the cerebellum in cognitive operations. Behav. Neurosci. 106, 731-738.

Albe-Fessard, D., Berkley, K. J., Kruger, L., Ralston, H. J. III, and Willis, W. D. Jr. (1985). Diencephalic mechanisms of pain sensation. Brain Res. 356, 217-296.

Allen, G., McColl, R., Barnard, H., Ringe, W. K., Fleckenstein, J., and Cullum, C. M. (2005). Magnetic resonance imaging of cerebellar-prefrontal and cerebellar-parietal functional connectivity. Neuroimage 28, 39-48.

Annett, M. (1970).A classification of hand preference by association analysis. $\mathrm{Br}$. J. Psychol. 61, 303-321.

Apkarian, A. V., Bushnell, M. C., Treede, R. D., and Zubieta, J. K. (2005). Human brain mechanisms of pain perception and regulation in health and disease. Eur. J. Pain 9, 463-484.

Baas, D., Aleman, A., and Kahn, R. S. (2004). Lateralization of amygdala activation: a systematic review of functional neuroimaging studies. Brain Res. Rev. 45, 96-103.

Becerra, L., Breiter, H. C., Wise, R., Gonzalez, R. G., and Borsook, D. (2001). Reward circuitry activation by noxious thermal stimuli. Neuron 32, 927-946

Bingel, U., Quante, M., Knab, R., Bromm, B., Weiller, C., and Büchel, C. (2002). Subcortical structures involved in pain processing: evidence from single-trial fMRI. Pain 99, 313-321.

Bingel, U., Quante, M., Knab, R., Bromm, B., Weiller, C., and Büchel, C. (2003). Single trial fMRI reveals significant contralateral bias in responses to laser pain within thalamus and somatosensory cortices. Neuroimage 18, 740-748.

Bornhovd, K., Quante, M., Glauche, V., Bromm, B., Weiller, C., and Büchel, C. (2002). Painful stimuli evoke different stimulus-response functions in the amygdala, prefrontal, insula and somatosensory cortex: a single-trial fMRI study. Brain 125(Pt 6), 1326-1336.

Borsook, D., Moulton, E. A., Tully, S., Schmahmann, J. D., and Becerra, L. (2008). Human cerebellar responses to brush and heat stimuli in healthy and neuropathic pain subjects. Cerebellum 7, 252-272.

Brooks, J. C., Nurmikko, T. J., Bimson, W. E., Singh, K. D., and Roberts, N. (2002). fMRI of thermal pain: effects of stimulus laterality and attention. Neuroimage 15, 293-301.

Brooks, J. C., Zambreanu, L., Godinez, A., Craig, A. D., and Tracey, I. (2005). Somatotopic organisation of the human insula to painful heat studied with high resolution functional imaging. Neuroimage 27, 201-209.

Büchel, C., Bornhovd, K., Quante, M., Glauche, V., Bromm, B., and Weiller, C. (2002). Dissociable neural responses related to pain intensity, stimulus intensity, and stimulus awareness within the anterior cingulate cortex: a parametric single-trial laser functional magnetic resonance imaging study. J. Neurosci. 22, 970-976.

Büchel, C., and Dolan, R. J. (2000). Classical fear conditioning in functional neuroimaging. Curr. Opin. Neurobiol. 10, 219-223.

Büchel, C., Dolan, R. J., Armony, J. L., and Friston, K. J. (1999). Amygdalahippocampal involvement in human aversive trace conditioning revealed through event-related functional magnetic resonance imaging. J. Neurosci. 19, 10869-10876.

Bukowska, D., Mierzejewska-Krzyzowska, B., and Zguczyński, L. (2006). Topography and axonal collaterals of trigeminocerebellar projection to the paramedian lobule and uvula in the rabbit cerebellum. Acta Neurobiol. Exp. (Wars) 66, 145-151.

Bushnell, M. C., and Duncan, G. H. (1989). Sensory and affective aspects of pain perception: is medial thalamus restricted to emotional issues? Exp. Brain Res. 78, 415-418.

Bushnell, M.C., Duncan, G.H., Hofbauer, R. K., Ha, B., Chen, J. I., and Carrier, B. (1999). Pain perception: is there a role for primary somatosensory cortex? Proc. Natl. Acad. Sci. U.S.A. 96, 7705-7709.

Cardinal, R. N., Parkinson, J. A., Lachenal, G., Halkerston, K. M., Rudarakanchana, N., Hall, J., Morrison, C. H., Howes, S. R., Robbins, T. W., and Everitt, B. J. (2002). Effects of selective excitotoxic lesions of the nucleus accumbens core, anterior cingulate cortex, and central nucleus of the amygdala on autoshaping performance in rats. Behav. Neurosci. 116, 553-567.

Christmann, C., Koeppe, C., Braus, D. F., Ruf, M., and Flor, H. (2007). A simultaneous EEG-fMRI study of painful electric stimulation. Neuroimage 34, 1428-1437.

Chudler, E. H. (1998). Response properties of neurons in the caudate-putamen and globus pallidus to noxious and non-noxious thermal stimulation in anesthetized rats. Brain Res. 812, 283-288.

Chudler, E. H., and Dong, W. K. (1995). The role of the basal ganglia in nociception and pain. Pain 60,3-38.

Cipolloni, P. B., and Pandya, D. N. (1999). Cortical connections of the frontoparietal opercular areas in the rhesus monkey. J. Comp. Neurol. 403, 431-458.

Coghill, R. C., Gilron, I., and Iadarola, M. J. (2001). Hemispheric lateralization of somatosensory processing. J. Neurophysiol. 85, 2602-2612.

Coghill, R. C., Sang, C. N., Maisog, J. M., and Iadarola, M. J. (1999). Pain intensity processing within the human brain: a bilateral, distributed mechanism. J. Neurophysiol. 82, 1934-1943.

Corbetta, M., and Shulman, G. L. (2002) Control of goal-directed and stimulusdriven attention in the brain. Nat. Rev Neurosci. 3, 201-202.

Craig, A. D. (2005). Forebrain emotional asymmetry: a neuroanatomical basis? Trends Cogn. Sci. 9, 566-571.

Crown, E. D., King, T. E., Meagher, M. W., and Grau, J. W. (2000). Shockinduced hyperalgesia: III. Role of the bed nucleus of the stria terminalis and amygdaloid nuclei. Behav. Neurosci. 114, 561-573.

Dietrichs, E., and Walberg, F. (1987) Cerebellar nuclear afferents - where do they originate? A re-evaluation of the projections from some lower brain stem nuclei. Anat. Embryol. 177, 165-172.

Dimitrova, A., Kolb, F. P., Elles, H.-G. Maschke, M., Forsting, M., Diener, H. C., and Timmann, D. (2003). Cerebellar responses evoked by nociceptive leg withdrawal reflex as revealed by event-related fMRI. J. Neurophysiol. 90, 1877-1886.

Disbrow, E., Roberts, T., and Krubitzer, L. (2000). Somatotopic organization of cortical fields in the lateral sulcus of Homo sapiens: evidence for SII and PV. J. Comp. Neurol. 418, 1-21.

Downar, J., Mikulis, D. J., and Davis, K. D. (2003). Neural correlates of the prolonged salience of painful stimulation. Neuroimage 20, 1540-1551.

Eickhoff, S. B., Amunts, K., Mohlberg, H. and Zilles, K. (2006). The human parietal operculum. II. Stereotaxic maps and correlation with functional imaging results. Cereb. Cortex 16, 268-279.

Eickhoff, S. B., Grefkes, C., Zilles, K., and Fink, G. R. (2007). The somatotopic organization of cytoarchitectonic areas on the human parietal operculum. Cereb. Cortex 17, 1800-1811.

Ettlin, D. A., Brügger, M., Keller, T., Luechinger, R., Jäncke, L., Palla, S., Barlow, A., Gallo, L. M., and Lutz, K. (2009). Interindividual differences in the perception of dental stimulation and related brain activity. Eur. J. Oral Sci. 1, 27-33.

Ettlin, D. A., Zhang, H., Lutz, K., Jarmann, T., Meier, D., Gallo, L. M., Jancke, L. and Palla, S. (2004). Cortical activation resulting from painless vibrotactile dental stimulation measured by func- tional magnetic resonance imaging (fMRI). J. Dent. Res. 83, 757-761.

Evans, A. J., Iwai, F., Grist, T. A., Sostman, H. D., Hedlund, L. W., Spritzer, C. E., Negro-Vilar, R., Beam, C. A., and Pelc, N. J. (1993). Magnetic resonance imaging of blood flow with a phase subtraction technique: in vitro and in vivo validation. Invest. Radiol. 28, 109-115.

Farrell, M. J., Laird, A. R., and Egan, G. F. (2005). Brain activity associated with painfully hot stimuli applied to the upper limb: a meta-analysis. Hum. Brain Mapp. 25, 129-139.

Farrer, C., Frey, S.H., van Horn, J.D., Tunik, E., Turk, D., Inati, S., and Grafton, S. T. (2008). The angular gyrus computes action awareness representations. Cereb. Cortex 18, 254-261.

Fillingim, R. B., and Ness, T. J. (2000). Sex-related hormonal influences on pain and analgesic responses. Neurosci. Biobehav. Rev. 24, 485-501.

Giesecke, T., Gracely, R. H., Grant, M. A., Nachemson, A., Petzke, F., Williams, D. A., and Clauw, D. J. (2004). Evidence of augmented central pain processing in idiopathic chronic low back pain. Arthritis Rheum. 50, 613-623.

Harvey, A. K., Pattinson, K. T. S., Brooks, J.C. W., Mayhew, S. D., Jenkinson, M. and Wise, R. G. (2008). Brainstem functional magnetic resonance imaging: disentangling signal from physiological noise. J. Magn. Reson. Imaging 28, 1337-1344.

Holtzman, T., Rajapaksa, T., Mostofi, A., and Edgley, S. A. (2006). Different responses of rat cerebellar Purkinje cells and Golgi cells evoked by widespread convergent sensory inputs. J. Physiol. (Lond.) 574(Pt 2), 491-507.

Ito, M. (2008). Control of mental activities by internal models in the cerebellum. Nat. Rev. Neurosci. 9, 304-313.

Jantsch, H. H., Kemppainen, P., Ringler, R., Handwerker, H. O., and Forster, C. (2005). Cortical representation of experimental tooth pain in humans. Pain 18, 390-399.

Jung, P., Baumgärtner, U., Bauermann, T., Magerl, W., Gawehn, J., Stoeter, P., and Treede, R.-D. (2003). Asymmetry in the human primary somatosensory cortex and handedness. Neuroimage 19, 913-923.

Keller, T., Popovic, M. R., Pappas, I. P., and Muller, P. Y. (2002). Transcutaneous functional electrical stimulator "Compex Motion". Artif. Organs 26, 219-223.

Kemppainen, P., Avellan, N. L., Handwerker, H. O., and Forster, C. (2003). Differences between tooth stimulation and capsaicin-induced neurogenic vasodilatation in human gingiva. J. Dent. Res. 82, 303-307. 
Kenshalo, D. R. Jr., Chudler, E. H., Anton, F., and Dubner, R. (1988). SI nociceptive neurons participate in the encoding process by which monkeys perceive the intensity of noxious thermal stimulation. Brain Res. 454, 378-382.

Kulkarni, B., Bentley, D. E., Elliott, R., Youell, P., Watson, A., Derbyshire, S. W., Frackowiak, R. S., Friston, K. J., and Jones, A. K. (2005). Attention to pain localization and unpleasantness discriminates the functions of the medial and lateral pain systems. Eur. J. Neurosci. 21, 3133-3142.

Lancaster, J. L., Summerln, J. L., Rainey, L. Freitas, C. S., and Fox, P.T. (1997). The Talairach Daemon, a database server for Talairach atlas labels. Neuroimage 5,633 .

Lancaster, J. L., Woldorff, M. G., Parsons, L. M., Liotti, M., Freitas, C. S., Rainey, L., Kochunov, P. V., Nickerson, D. Mikiten, S. A., and Fox, P. T. (2000). Automated Talairach atlas labels for functional brain mapping. Hum. Brain Mapp. 10, 120-131.

Legrain, V., van Damme, S., Eccleston, C., Davis, K. D., Seminowicz, D. A., and Crombez, G. (2009). A neurocognitive model of attention to pain: behavioral and neuroimaging evidence. Pain 144, 230-232.

Maldjian, J. A., Laurienti, P. J., Kraft, R. A., and Burdette, J. H. (2003). An automated method for neuroanatomic and cytoarchitectonic atlas-based interrogation of fMRI data sets. Neuroimage 19, 1233-1239.

Manto, M.-U. (2006). On the cerebellocerebral interactions. Cerebellum 5, 286-288.

Mouraux, A., and Iannetti, G. D. (2009). Nociceptive laser-evoked brain potentials do not reflect nociceptive-specific neural activity. J. Neurophysiol. 101, 3258-3269.

Neugebauer, V., and Li, W. (2002). Processing of nociceptive mechanical and thermal information in central amygdala neurons with knee-joint input. J. Neurophysiol. 87, 103-112.

Neugebauer, V., and Li, W. (2003). Differential sensitization of amygdala neurons to afferent inputs in a model of arthritic pain. J. Neurophysiol. 89, 716-727.

Neugebauer, V., Li, W., Bird, G. C., and Han, J. S. (2004). The amygdala and persistent pain. Neuroscientist 10, 221-234.

Patrick, G. W., and Robinson, M. A. (1987). Collateral projections from trigeminal sensory nuclei to ventrobasal thalamus and cerebellar cortex in rats. J. Morphol. 192, 229-236.

Petrovic, P., Petersson, K. M., Ghatan, P. H., Stone-Elander, S., and Ingvar, M. (2000). Pain-related cerebral activation is altered by a distracting cognitive task. Pain 85, 19-30.

Peyron, R., Kupers, R., Jehl, J. L., GarciaLarrea, L., Convers, P., Barral, F. G., and Laurent, B. (2007). Central representation of the RIII flexion reflex associated with overt motor reaction: an fMRI study. Clin. Neurophysiol. 37, 249-259.

Peyron, R., Laurent, B., and Garcia-Larrea, L. (2000). Functional imaging of brain responses to pain: a review and metaanalysis (2000). Neurophysiol. Clin. 30, 263-288.

Phan, K. L., Taylor, S. F., Welsh, R. C., Ho, S.-H., Britton, J. C., and Liberzon, I. (2004). Neural correlates of individual ratings of emotional salience: a trialrelated fMRI study. Neuroimage 21, 768-780.

Ploghaus, A., Tracey, I., Clare, S., Gati, J. S., Rawlins, J. N., and Matthews, P. M. (2000). Learning about pain: the neural substrate of the prediction error for aversive events. Proc. Natl. Acad. Sci. U.S.A. 97, 9281-9286.

Rempel-Clower, N. L. (2007). Role of orbitofrontal cortex connections in emotion. Ann. N. Y. Acad. Sci. 1121, 72-86.

Saab, C. Y., Kawasaki, M., Al-Chaer, E. D., and Willis, W. D. (2001). Cerebellar cortical stimulation increases spinal visceral nociceptive responses. J. Neurophysiol. 85, 2359-2363.

Saab, C. Y., and Willis, W. D. (2003). The cerebellum: organization, functions and its role in nociception. Brain Res. 42, 85-95.

Schlereth, T., Baumgärtner, U., Magerl,W., Stoeter, P., and Treede, R.-D. (2003). Left-hemisphere dominance in early nociceptive processing in the human parasylvian cortex. Neuroimage 20, 441-454.

Schmahmann, J.D., and Caplan, D. (2006). Cognition, emotion and the cerebellum. Brain 129(Pt 2), 290-292.
Schmahmann, J. D., and Pandya, D. N. (1997). The cerebrocerebellar system. Int. Rev. Neurobiol. 41, 31-60.

Schoedel, A. L., Zimmermann, K. Handwerker, H. O., and Forster, C. (2008). The influence of simultaneous ratings on cortical BOLD effects during painful and non-painful stimulation. Pain 135, 131-141.

Scott, D. J., Heitzeg, M. M., Koeppe, R. A., Stohler, C. S., and Zubieta, J. K. (2006). Variations in the human pain stress experience mediated by ventral and dorsal basal ganglia dopamine activity. J. Neurosci. 26, 10789-10795.

Serrien, D. J., Ivry, R. B., and Swinnen, S. P. (2006). Dynamics of hemispheric specialization and integration in the context of motor control. Nat. Rev. Neurosci. 7, 160-166.

Sessle, B. J. (2000). Acute and chronic craniofacial pain: brainstem mechanisms of nociceptive transmission and neuroplasticity, and their clinical correlates. Crit. Rev. Oral Biol. Med. 11, 57-91.

Snyder, R. L., Faull, R. L., and Mehler, W R. (1978). A comparative study of the neurons of origin of the spinocerebellar afferents in the rat, cat and squirrel monkey based on the retrograde transport of horseradish peroxidase. J. Comp. Neurol. 181, 833-852.

Strange, B. A., and Dolan, R. J. (2006) Anterior medial temporal lobe in human cognition: memory for fear and the unexpected. Cogn. Neuropsychiatry 11, 198-218.

Symonds, L. L., Gordon, N. S., Bixby, J. C., and Mande, M. M. (2006). Right-lateralized pain processing in the human cortex: an fMRI study. J. Neurophysiol. 95, 3823-3830.

Tracey, I., and Mantyh, P. W. (2007). The cerebral signature for pain perception and its modulation. Neuron 55, 377-391.

Treede, R. D., Kenshalo, D. R., Gracely, R. H., and Jones, A. K. (1999). The cortical representation of pain. Pain 79, 105-111.

Varnavas, G. G., and Grand, W. (1999) The insular cortex: morphological and vascular anatomic characteristics. Neurosurgery 44, 127-136; discussion 136-138.

Vogt, B. A. (2005). Pain and emotion interactions in subregions of the cingulate gyrus. Nat. Rev. Neurosci. 6, 533-544.

Watkins, L. R., Wiertelak, E. P., McGorry, M., Martinez, J., Schwartz, B., Sisk, D., and Maier, S. F. (1998). Neurocircuitry of conditioned inhibition of analgesia: effects of amygdala, dorsal raphe, ventral medullary, and spinal cord lesions on antianalgesia in the rat. Behav. Neurosci. 112, 360-378.

Wiech, K., Kalisch, R., Weiskopf, N., Pleger, B., Stephan, K. E., and Dolan, R. J. (2006). Anterolateral prefrontal cortex mediates the analgesic effect of expected and perceived control over pain. J. Neurosci. 26, 11501-11509.

Wiesenfeld-Hallin, Z. (2005). Sex differences in pain perception. Gend. Med. 2, 137-145.

Worsley, K. J., Marrett, S., Neelin, P., Vandal, A. C., Friston, K. J., and Evans, A. C. (1996). A unified statistical approach for determining significant signals in images of cerebral activation. Hum. Brain Mapp. 4, 58-73.

Youell, P. D., Wise, R. G., Bentley, D. E., Dickinson, M. R., King, T.A., Tracey, I., and Jones, A. K. (2004). Lateralisation of nociceptive processing in the human brain: a functional magnetic resonance imaging study. Neuroimage 23, 1068-1077.

Conflict of Interest Statement: The authors declare that the research was conducted in the absence of any commercial or financial relationships that could be construed as a potential conflict of interest.

Received: 28 February 2010; paper pending published: 28 July 2010; accepted: 21 January 2011; published online: 07 February 2011.

Citation: Brügger M, Ettlin DA, Meier M, Keller T, Luechinger R, Barlow A, Palla S, Jäncke L and Lutz K (2011) Taking sides with pain - lateralization aspects related to cerebral processing of dental pain. Front. Hum. Neurosci. 5:12. doi: 10.3389/ fnhum.2011.00012

Copyright () 2011 Brügger, Ettlin, Meier, Keller, Luechinger, Barlow, Palla, Jäncke and Lutz. This is an open-access article subject to an exclusive license agreement between the authors and Frontiers Media SA, which permits unrestricted use, distribution, and reproduction in any medium, provided the original authors and source are credited. 


\section{APPENDIX}

Table A1 | Complete list of local maxima within clusters activated for the contrast stimulation versus baseline (as illustrated in Figure 2).

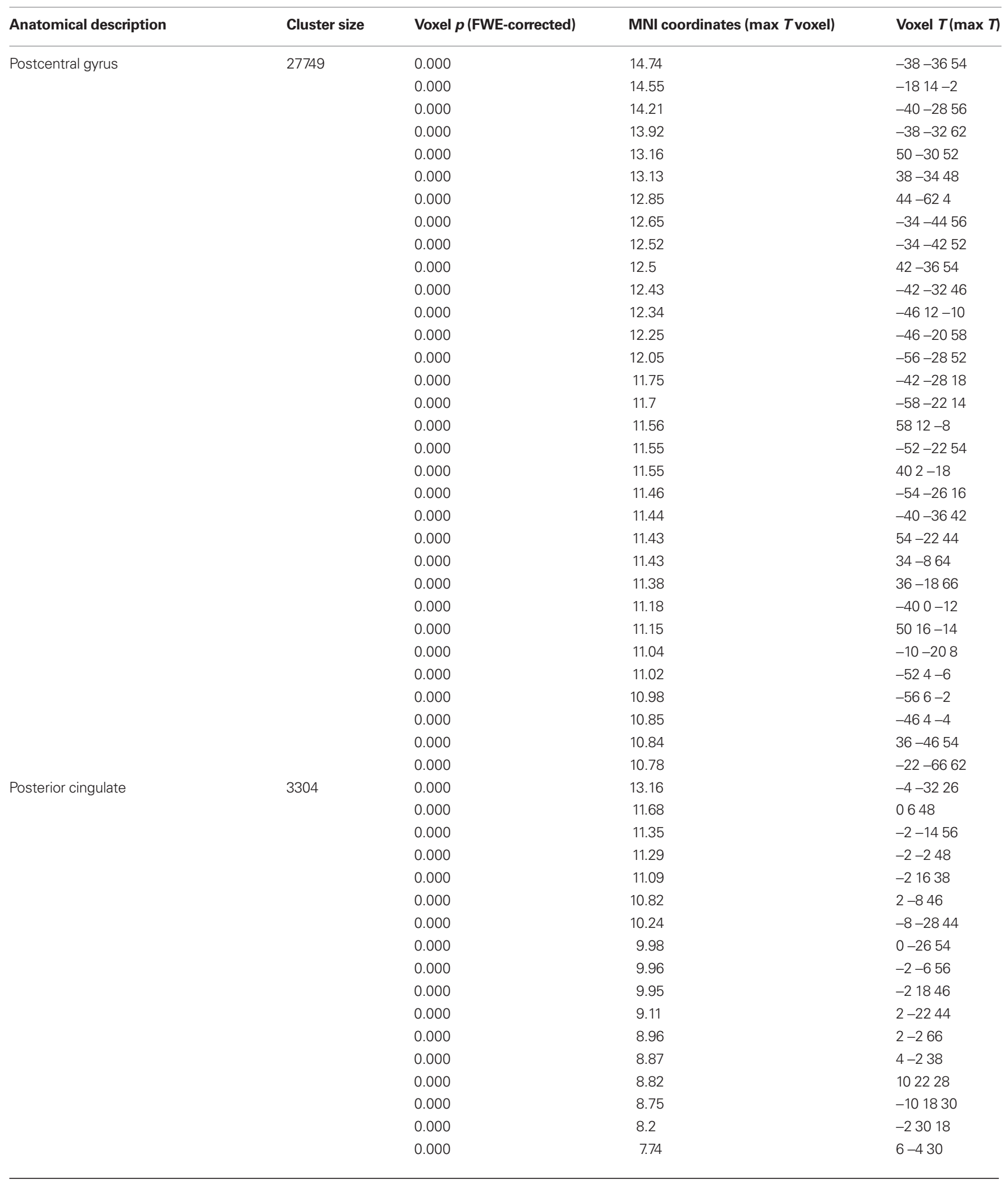


Table A1 | Continued

\begin{tabular}{|c|c|c|c|c|}
\hline Anatomical description & Cluster size & Voxel $p$ (FWE-corrected) & MNI coordinates (max $T$ voxel) & Voxel $T(\max T)$ \\
\hline & & 0.000 & 7.31 & $4-2028$ \\
\hline & & 0.000 & 6.86 & $2-1428$ \\
\hline & & 0.000 & 6.48 & -23810 \\
\hline & & 0.003 & 6.02 & 10440 \\
\hline & & 0.000 & 7.4 & $4-26-28$ \\
\hline & & 0.000 & 7.2 & $0-18-20$ \\
\hline Cerebellum posterior lobe & 117 & 0.000 & 9.6 & $14-76-48$ \\
\hline \multirow[t]{2}{*}{ Medulla } & 72 & 0.000 & 8.07 & $-2-34-50$ \\
\hline & & 0.000 & 6.48 & $-6-34-42$ \\
\hline \multirow{2}{*}{ Temporal inferior lobe } & & 0.000 & 6.51 & $-38-48-24$ \\
\hline & & 0.002 & 6.11 & $-48-42-26$ \\
\hline Cingulate gyrus & 13 & 0.000 & 6.79 & $14-2836$ \\
\hline \multirow[t]{2}{*}{ Occipital lobe (lingual) } & 55 & 0.000 & 6.69 & $2-684$ \\
\hline & & 0.000 & 6.53 & $6-640$ \\
\hline \multirow[t]{2}{*}{ Parietal lobe (precuneus) } & 17 & 0.000 & 6.62 & $-6-5252$ \\
\hline & & 0.001 & 6.25 & $-2-5852$ \\
\hline Inferior parietal lobe (supramarginal) & 11 & 0.001 & 6.2 & $68-3626$ \\
\hline Only & & & & \\
\hline
\end{tabular}

As there are very large clusters, anatomical descriptions are related only to the maximally activated voxel within each cluster. 